Received: 9 February 2018

Accepted: 21 May 2018

Published online: 11 June 2018
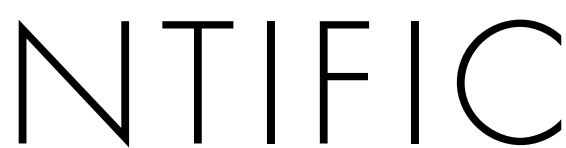

REP

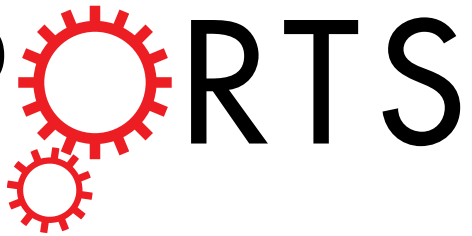

OPEN

\section{Thermally Activated Delayed Fluorescence Host for High Performance Organic Light- Emitting Diodes}

\section{Lu Zhang \& Kok Wai Cheah}

Thermally activated delayed florescence (TADF) materials can be an efficient host in organic LED (OLED). It is because it is possible to couple energetically the emission energy level of a dopant to the energy levels in the TADF material. In this work fluorescent emitters 2,3,6,7-tetrahydro1,1,7,7,-tetramethyl-1H,5 H,11H-10-(2-benzothiazolyl)quinolizino-9,9a,1gh coumarin (c545t) and 5,6,11,12-tetraphenyltetracene (rubrene) were used as dopants in a blended TADF host; tris(4carbazoyl-9-ylphenyl)amine (TCTA) with 2,4,6-tris(3'-(pyridin-3-yl)biphenyl-3-yl)-1,3,5-triazine (Tm3PyBPZ). The blended TADF host has an energy difference between the singlet and triplet excited states $\left(\Delta E_{S T}\right)$ around $27 \mathrm{meV}$ with the yield of reverse intersystem crossing $\left(\Phi_{\mathrm{RISC}}\right)$ nearly $100 \%$. This high $\Phi_{\text {RISC }}$ yield enhances the OLED performance with the c545t doped OLED having $11.9 \%$ external quantum efficiency and $10 \%$ for the rubrene doped OLED.

When an organic light-emitting diode (OLED) is working under the electric field, the holes and electrons are injected from the electrodes and then form singlet excitons and triplet excitons in the emissive layer (EML) with the ratio of approximately $25 \%: 75 \%$ from spin statistics. In a conventional fluorescent OLED, the $75 \%$ triplet excitons are generally non-emissive at room temperature, which limits the internal quantum efficiency (IQE) to be only $25 \%$. By introducing heavy-metal induced spin-orbit coupling, the transitions between singlet and triplet states are possible which makes it possible to utilize both singlet and triplet excitons ${ }^{1}$. However, these transition metals cause other concerns such as high cost and environmental pollution. In recent years, a new approach to efficiently utilize the triplet excitons generated in the OLED has drawn a lot of attention, which is thermally activated delayed fluorescence (TADF); metal-free TADF fluorescent materials can harvest both singlet and triplet excitons, which greatly improves the IQE compared to the conventional fluorescent materials ${ }^{2}$. For a TADF material, the energy difference between singlet excited state and triplet excited state $\left(\Delta \mathrm{E}_{\mathrm{ST}}\right)$ is usually very small $(<0.1 \mathrm{eV})$. Therefore, the triplet excitons can be converted to singlet excitons via reverse intersystem crossing (RISC) with the assistance of thermal energy, which makes it possible to realize $100 \%$ IQE without using heavy metals ${ }^{3}$.

A small $\Delta \mathrm{E}_{\mathrm{ST}}$ is required for a TADF material, which enables a high RISC rate constant $\left(\mathrm{k}_{\mathrm{RISC}}\right)$ according to the equation as follows:

$$
k_{\text {RISC }}=A \exp \left(-\frac{\Delta E_{S T}}{k_{B} T}\right)
$$

where $\mathrm{A}$ is a constant, $\mathrm{k}_{\mathrm{B}}$ is the Boltzmann constant, and $\mathrm{T}$ is the temperature ${ }^{4}$. Therefore, the RISC can efficiently compete with other non-radiative decay such as internal conversion (IC). Since $\Delta \mathrm{E}_{\mathrm{ST}}$ scales with the spatial overlap of the highest occupied molecular orbital (HOMO) and lowest unoccupied molecular orbital (LUMO) wave functions $\Psi_{\mathrm{HOMO}}$ and $\Psi_{\mathrm{LUMO}}$ as shown in equation (2), the HOMO and LUMO of TADF materials are designed to be separately located on donor moiety and acceptor moiety, respectively, which results in a small $\Delta \mathrm{E}_{\mathrm{ST}}$ while maintaining a high fluorescent quantum yield ${ }^{5,6}$.

Department of Physics and Institute of Advanced Materials, Hong Kong Baptist University, Kowloon Tong, Hong Kong SAR, China. Correspondence and requests for materials should be addressed to K.W.C. (email: kwcheah@ hkbu.edu.hk) 


\begin{tabular}{|l|l|l|l|l|l|l|}
\hline Temperature $(\mathbf{K})$ & $\mathbf{A}_{\mathbf{p}}$ & $\boldsymbol{\tau}_{\mathbf{p}}(\mathbf{n s})$ & $\mathbf{A}_{\mathbf{d}}$ & $\boldsymbol{\tau}_{\mathbf{d}}(\boldsymbol{\mu s})$ & $\boldsymbol{\Phi}_{\mathbf{d}} / \boldsymbol{\Phi}_{\mathbf{p}}$ & $\mathbf{k}_{\text {RISC }}\left(\mathbf{s}^{-1}\right)$ \\
\hline 205 & 0.1467 & 20.7 & 0.0100 & 2.3 & 7.68 & $3.7 \times 10^{6}$ \\
\hline 215 & 0.1599 & 18.0 & 0.0098 & 2.2 & 7.41 & $3.9 \times 10^{6}$ \\
\hline 225 & 0.1506 & 18.5 & 0.0097 & 2.1 & 7.36 & $4.0 \times 10^{6}$ \\
\hline 235 & 0.1202 & 20.7 & 0.0096 & 2.0 & 7.69 & $4.4 \times 10^{6}$ \\
\hline 245 & 0.1113 & 19.7 & 0.0086 & 1.8 & 6.96 & $4.5 \times 10^{6}$ \\
\hline 255 & 0.0975 & 20.8 & 0.0085 & 1.7 & 6.97 & $4.8 \times 10^{6}$ \\
\hline 265 & 0.0847 & 22.0 & 0.0081 & 1.6 & 6.89 & $5.0 \times 10^{6}$ \\
\hline 275 & 0.0798 & 21.7 & 0.0077 & 1.4 & 6.35 & $5.1 \times 10^{6}$ \\
\hline 285 & 0.0582 & 22.2 & 0.0062 & 1.3 & 6.22 & $5.6 \times 10^{6}$ \\
\hline 295 & 0.0428 & 24.7 & 0.0054 & 1.2 & 6.40 & $6.0 \times 10^{6}$ \\
\hline
\end{tabular}

Table 1. The time-resolved PL characteristics, the ratio between DF and PF $\left(\Phi_{d} / \Phi_{p}\right)$ and the RISC rate constant $\left(\mathrm{k}_{\mathrm{RISC}}\right)$ of a TCTA:Tm3PyBPZ $(1: 1,50 \mathrm{~nm})$ film at different temperatures.

$$
\Delta E_{S T} \propto \int \Psi_{H O M O}(r) \Psi_{L U M O}(r) d_{r}^{3}
$$

A small $\Delta \mathrm{E}_{\mathrm{ST}}$ can also be realized in some intermolecular excited-state complex (exciplex) formed between electron-donating molecule and electron-accepting molecule ${ }^{7,8}$. Therefore, they are good candidates for triplet harvesting through RISC. In an exciplex exhibiting TADF, the HOMO and LUMO are mainly located on donor and acceptor molecules, respectively. This kind of TADF material can be functioned as the emitter or as the host for some conventional fluorescent emitter to improve the IQE ${ }^{9,10}$.

\section{Results}

In our earlier work, we have demonstrated efficient TADF OLEDs based on the exciplex forming between electron-donating material tris(4-carbazoyl-9-ylphenyl)amine (TCTA) and electron-accepting material 2,4,6-tris (3'-(pyridin-3-yl)biphenyl-3-yl)-1,3,5-triazine (Tm3PyBPZ) ${ }^{11}$. The exciplex shows a green emission with the peak wavelength around $514 \mathrm{~nm}$. The maximum efficiencies of OLEDs based on TCTA:Tm3PyBPZ (1:1) are $13.1 \%$ and $53.4 \mathrm{~lm} / \mathrm{W}$ under low turn-on voltage of only $2.4 \mathrm{~V}$.

Here we estimate the $\Delta \mathrm{E}_{\mathrm{ST}}$ of TCTA:Tm3PyBPZ; time-resolved photoluminescence (PL) characteristics of TCTA:Tm3PyBPZ (1:1, $50 \mathrm{~nm}$ ) film from $205 \mathrm{~K}$ to $295 \mathrm{~K}$ were measured by exciting the sample with a pulsed Nd:YAG laser. Since the emission of TADF material consists of prompt fluorescence (PF) that happened immediately after the excitation and delayed fluorescence (DF) through the RISC, the time-resolved PL characteristics were fitted by bi-exponential decay as shown in equation (3):

$$
I=A_{p} e^{-\frac{t}{\tau_{p}}}+A_{d} e^{-\frac{t}{\tau_{d}}}
$$

where $A_{p}$ and $A_{d}$ are the proportional quantities of PF and DF, respectively. $\tau_{p}$ and $\tau_{d}$ are the lifetimes of PF and DF, respectively. The ratio between DF and $P F\left(\Phi_{d} / \Phi_{p}\right)$ at different temperatures can be determined by the integral of the DF and PF components according to equation (4). The fitting results and the calculated $\Phi_{\mathrm{d}} / \Phi_{\mathrm{p}}$ are shown in Table 1.

$$
\frac{\Phi_{d}}{\Phi_{p}}=\frac{A_{d} \tau_{d}}{A_{p} \tau_{p}}
$$

The fluorescence yield of TADF emitters $\left(\Phi_{\mathrm{F}}\right)$ is described by equation (5), which includes recycling of singlet and triplet states.

$$
\Phi_{F}=\Phi_{p}+\Phi_{d}=\sum_{i=0}^{n} \Phi_{p}\left(\Phi_{I S C} \Phi_{R I S C}\right)^{i}=\Phi_{p} \frac{1}{1-\Phi_{I S C} \Phi_{R I S C}}
$$

Therefore, $\Phi \mathrm{d} / \Phi \mathrm{p}$ can be expressed in terms of $\Phi_{\mathrm{ISC}}$ and $\Phi_{\mathrm{RISC}}$ as shown in equation (6).

$$
\frac{\Phi_{d}}{\Phi_{p}}=\frac{1}{1-\Phi_{I S C} \Phi_{R I S C}}-1
$$

For $\Phi \mathrm{d} / \Phi \mathrm{p} \approx 4, \Phi_{\mathrm{ISC}} \Phi_{\mathrm{RISC}}$ is around 0.8 . Since $\Phi_{\mathrm{ISC}}{ }^{\max }=1-\Phi \mathrm{p}$, therefore $\Phi_{\mathrm{RISC}}$ is close to $1^{12,13}$. In that case, the rate of RISC $\left(\mathrm{k}_{\mathrm{RISC}}\right)$ can be simply expressed by equation (7):

$$
k_{\text {RISC }}=\frac{1}{\tau_{d}}\left(\frac{\Phi_{p}+\Phi_{d}}{\Phi_{p}}\right)
$$

Thus, $\mathrm{k}_{\text {RISC }}$ can be derived from $\Phi_{\mathrm{d}} / \Phi_{\mathrm{p}}$ and $\tau_{\mathrm{d}}{ }^{[12]}$ In this work, $\Phi_{\mathrm{d}} / \Phi_{\mathrm{p}}$ was obtained through equations (3) and (4) and is above 6 as shown in Table 1; thus the $\mathrm{k}_{\mathrm{RISC}}$ can be calculated from equation (7) and shown in Table 1 . When 


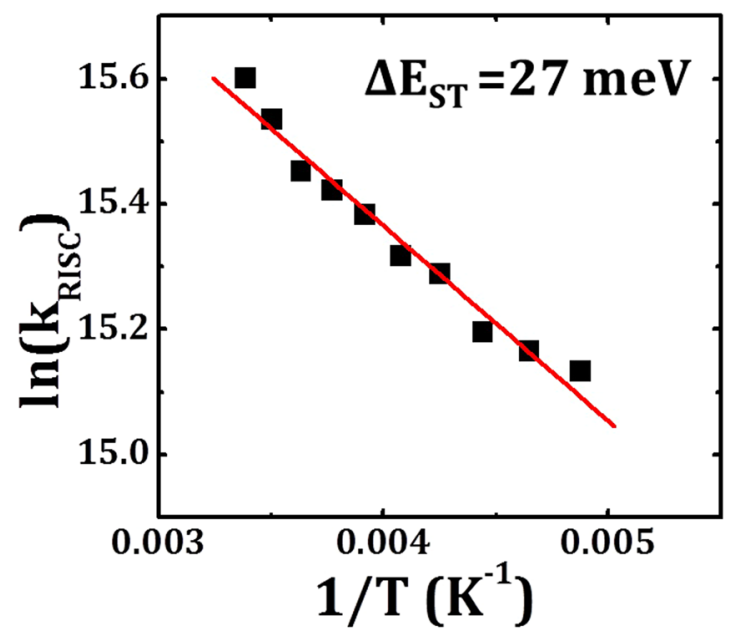

Figure 1. Arrhenius plot of the RISC rate constant of TCTA:Tm3PyBPZ (1:1, 50 nm) film. The square root of mean square error is 0.029 .

(a)

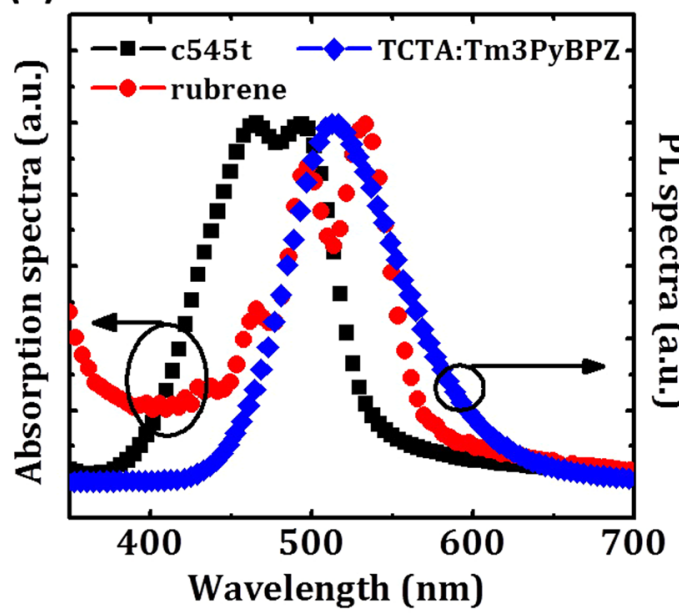

(b)



Figure 2. (a) absorption spectra of c545t and rubrene and emission spectrum of exciplex TADF host based on TCTA:Tm3PyBPZ (1:1) in solid state. (b) PL spectra of c545t and rubrene in solid state.

the temperature was decreased from $295 \mathrm{~K}$ to $205 \mathrm{~K}, \mathrm{k}_{\mathrm{RISC}}$ decreased from $6.0 \times 10^{6} \mathrm{~s}^{-1}$ to $3.7 \times 10^{6} \mathrm{~s}^{-1}$. Since $\mathrm{k}_{\mathrm{RISC}}$ is related to $\Delta \mathrm{E}_{\mathrm{ST}}$ and temperature (equation 1), the activation energy can be estimated to be $27 \mathrm{meV}$ from the Arrhenius plot as shown in Fig. 1.

The TADF material can also function as host for conventional fluorescent emitter improving the IQE. As shown in Fig. S1, the triplet excitons formed at the exciplex host can be converted to singlet excitons by RISC. Then the additional energy is transferred from singlet energy level of the host to the singlet energy level of the fluorescent emitter to increase the singlet excitons population formed on emitter.

OLEDs were fabricated using TCTA:Tm3PyBPZ (1:1) exciplex as host for 2,3,6,7-tetrahydro-1,1,7,7,-tetramethyl$1 \mathrm{H}, 5 \mathrm{H}, 11 \mathrm{H}-10$-(2-benzothiazolyl) quinolizino-9,9a,1gh coumarin (c545t) and 5,6,11,12-tetraphenyltetracene (rubrene). The chemical structures of TCTA, Tm3PyBPZ, c545t and rubrene are shown in Fig. S2. The energy level diagrams are shown in Fig. S3. The HOMO and LUMO levels of c545t and rubrene are near to those of the exciplex which minimize the exciton trapping and facilitate the transfer of excitation energy ${ }^{14-16}$. The absorption spectra of the dopants are well overlapped with the emission of the exciplex host as shown in Fig. 2a, which enables efficient Förster energy transfer from the host to the dopants. Figure $2 b$ shows the PL spectra of thin films of c545t $(50 \mathrm{~nm})$ and rubrene $(50 \mathrm{~nm})$. The peak wavelengths of c545t and rubrene are $564 \mathrm{~nm}$ and $563 \mathrm{~nm}$, respectively.

The OLED devices with a structure of 1,4,5,8,9,11-hexaazatriphenylenehexacarbonitrile $\left[\mathrm{Hat}(\mathrm{CN})_{6}\right.$, $5 \mathrm{~nm}$ ]/1,1-bis(4-(N,N-di(p-tolyl)-amino)phenyl)cyclohexane [TAPC, $65 \mathrm{~nm}$ ]/EML [10 nm]/Tm3PyBPZ [50 nm]/8-hydroxy-quinolinato lithium [Liq, $2 \mathrm{~nm}] / \mathrm{Al}[100 \mathrm{~nm}]$ were fabricated onto indium tin oxide (ITO) glass substrate (sheet resistance $\sim 18 \Omega$ /square). For the EML, the host was exciplex forming by co-evaporating TCTA and Tm3PyBPZ with the ratio of 1:1. Four OLEDs were fabricated: for Device A and B, the EML were c545t 

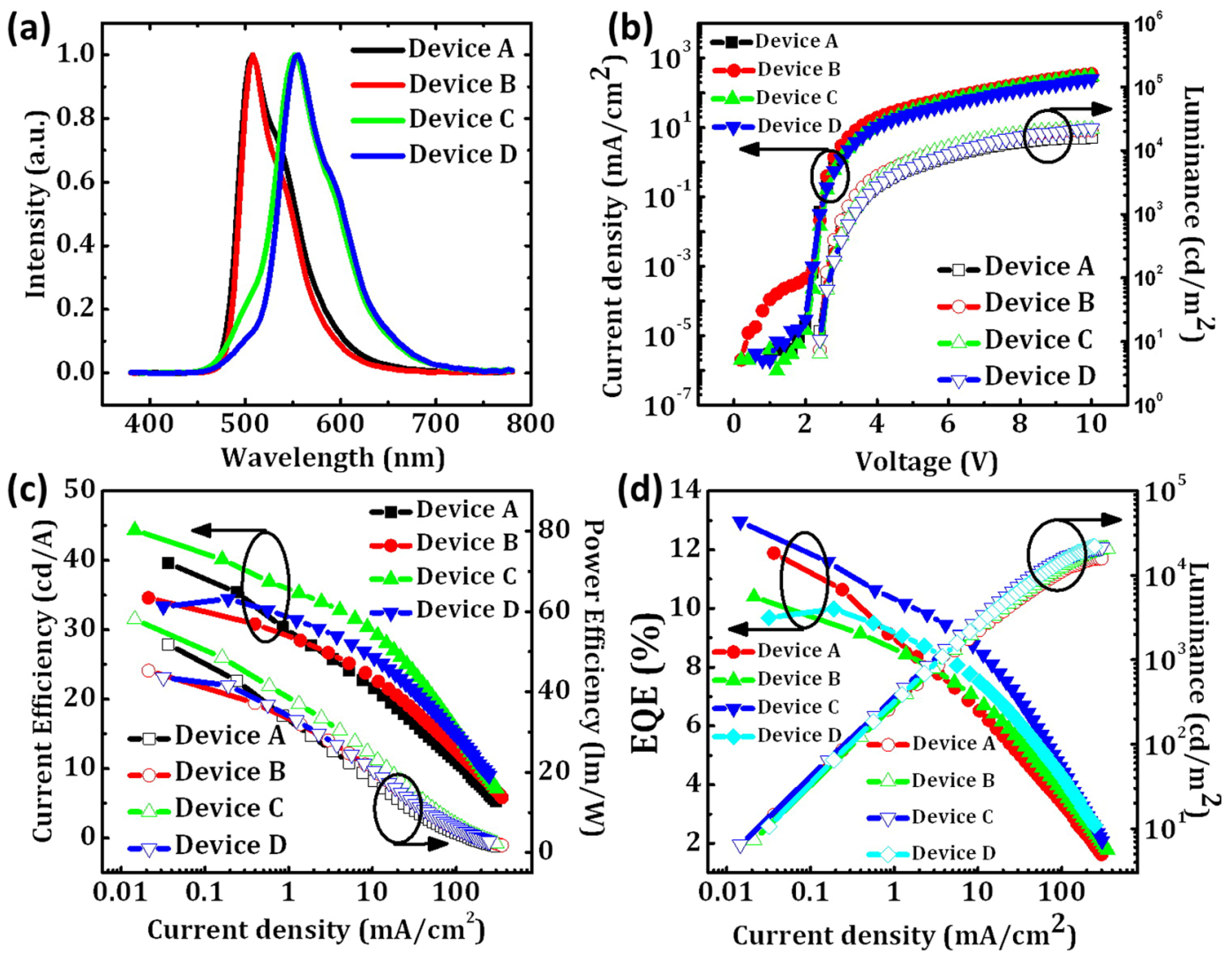

Figure 3. (a) EL spectra of the OLED devices. (b) current density and luminance versus voltage characteristics. (c) current efficiency and power efficiency versus current density characteristics. (d) EQE and luminance versus current density characteristics.
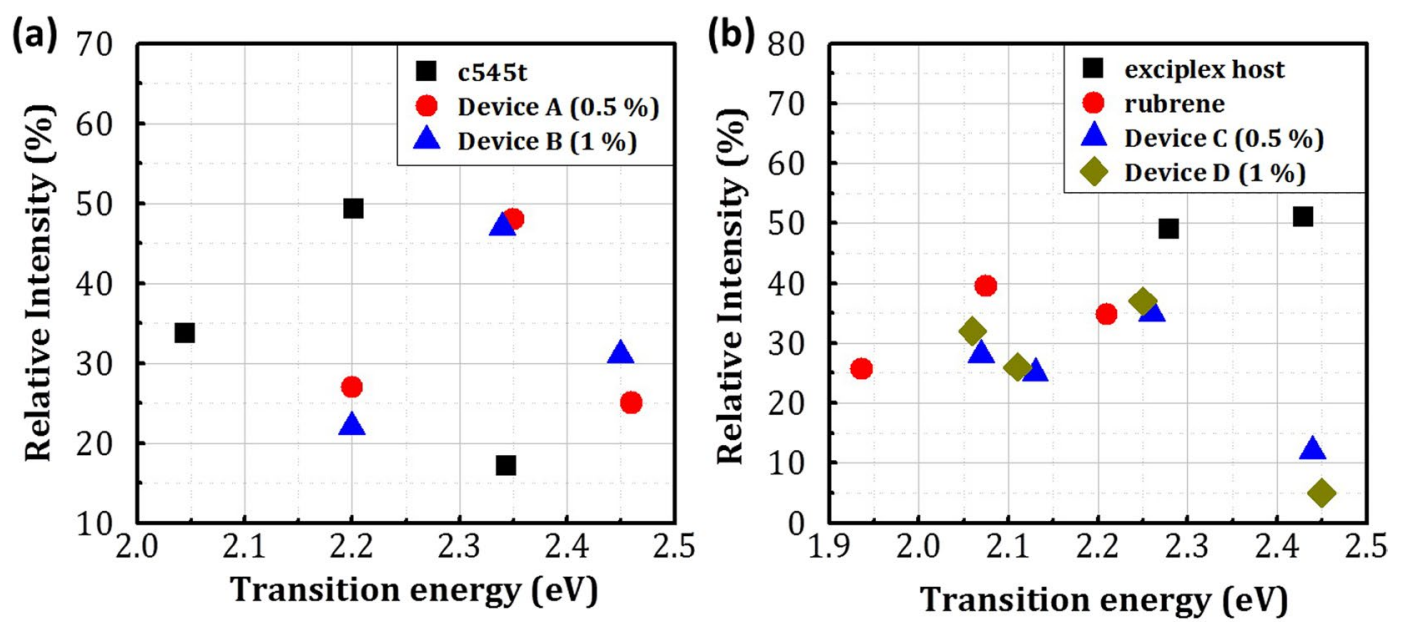

Figure 4. Transition energies and the corresponding relative intensities of multi-peaks fitting results using a Gaussian function. (a) Gaussian fitting results of PL spectrum of c545t thin film and EL spectra of c545t based OLEDs. (b) Gaussian fitting results of PL spectra of exciplex host and rubrene thin film and EL spectra of rubrene based OLEDs.

doped with host and the concentrations are $0.5 \%$ and $1 \%$, respectively; for Device C and D, the EML were rubrene doped with host and the concentrations are $0.5 \%$ and $1 \%$, respectively. Low doping concentrations were used in the EML to reduce the triplet-triplet energy transfer from the host to the dopants.

The electroluminescence (EL) spectra of all the devices are shown in Fig. 3a. We analyzed the PL spectra (Fig. 2) and EL spectra (Fig. 3a) with a multi-peak fitting using a Gaussian function (Fig. S4) and the derived parameters are shown in Fig. 4 and Table S1. From this analysis, we have determined the PL spectra to have three 
(a) $\mathrm{s}_{1}$

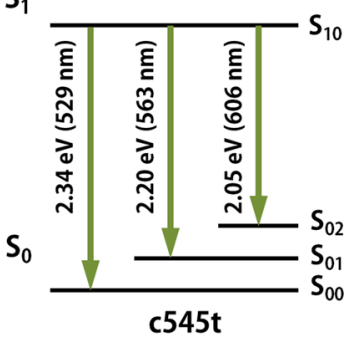

(b) $S_{1}$

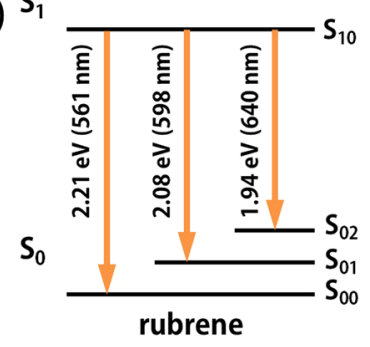

Figure 5. Energy level scheme of c545t and rubrene as deduced from the PL spectra.

\begin{tabular}{|c|c|c|c|c|c|c|c|c|c|c|c|}
\hline \multirow[b]{3}{*}{ Device } & \multicolumn{4}{|c|}{ Maximum } & \multicolumn{3}{|c|}{ at $100 \mathrm{~cd} / \mathrm{m}^{2}$} & \multicolumn{3}{|c|}{ at $1000 \mathrm{~cd} / \mathrm{m}^{2}$} & \multirow[b]{3}{*}{$\operatorname{CIE}(x, y)$} \\
\hline & $\mathbf{L}$ & $\mathrm{CE}$ & PE & EQE & CE & PE & EQE & CE & PE & EQE & \\
\hline & $\mathrm{cd} / \mathrm{m}^{2}$ & cd/A & $\operatorname{lm} / \mathrm{W}$ & $\%$ & $\mathrm{~cd} / \mathrm{A}$ & $\operatorname{lm} / \mathrm{W}$ & $\%$ & $\mathrm{~cd} / \mathrm{A}$ & $\operatorname{lm} / \mathrm{W}$ & $\%$ & \\
\hline A & 15870 & 39.6 & 51.9 & 11.9 & 35.4 & 42.8 & 10.6 & 25.8 & 25.3 & 7.8 & $(0.25,0.62)$ \\
\hline B & 20640 & 34.6 & 45.3 & 10.4 & 30.8 & 37.3 & 9.1 & 26.7 & 28.0 & 7.9 & $(0.24,0.64)$ \\
\hline $\mathrm{C}$ & 21620 & 44.4 & 58.1 & \begin{tabular}{|l|}
13.0 \\
\end{tabular} & 40.2 & 48.5 & 11.6 & 34.0 & 33.4 & 9.8 & $(0.41,0.55)$ \\
\hline $\mathrm{D}$ & 22170 & 34.5 & 41.6 & 10.0 & 34.5 & 41.6 & 10.0 & 29.1 & 26.9 & 8.4 & $(0.44,0.54)$ \\
\hline
\end{tabular}

Table 2. Luminance (L), current efficiencies (CE), power efficiencies (PE) and external quantum efficiencies (EQE) of the OLED devices.

transitions in c545t and rubrene ${ }^{17}$. The established energy levels are shown in Fig. 5. $\mathrm{S}_{0}$ and $\mathrm{S}_{1}$ indicate a series of singlet states which further subdivided into various vibrational states representing by adding a second subscript. As shown in Fig. 4a, the transitions in the c545t thin film were found to have the energies of $2.34 \mathrm{eV}, 2.2 \mathrm{eV}$ and $2.05 \mathrm{eV}$. These transitions have lower energies compared with those in the OLEDs since the distance between dopant molecules has increased so the $\pi-\pi$ overlap is reduced and result in a blueshift of the spectra. No transition from the exciplex host is observed in both c545t based devices (Fig. S4d and e) indicating a good Förster energy transfer from the host to the dopant. The transitions in the rubrene thin film were found to have the energies of $2.21 \mathrm{eV}, 2.08 \mathrm{eV}$ and $1.94 \mathrm{eV}$ (Fig. 5b). However, both rubrene based devices showed the emission from the exciplex host $(\sim 2.45 \mathrm{eV})$ as well as the three transitions from rubrene as shown in Fig. 4b. The appearance of exciplex emission is due to incomplete energy transfer from the host to the dopant. This is evident from the fact that the relative intensity of the emission from the host is reduced from $12 \%$ to $5 \%$ when the doping concentration increasing from $0.5 \%$ to $1 \%$ since the decreasing of the distance between host and dopant results in a higher rate constant of Förster energy transfer.

The current density and luminance versus voltage characteristics is shown in Fig. 3b. All the devices show very low turn-on voltage of $2.4 \mathrm{~V}$. It is likely that the additional current appeared in device B is probably caused by the thermally activated leakage associated with device imperfections as well as the drift of the residual charge carrier in the intrinsic organic material when it is biased ${ }^{18,19}$. For green OLED devices based on c545t, Device A with the doping concentration of $0.5 \%$ shows higher external quantum efficiency (EQE), power efficiency (PE) and current efficiency (CE) when the current density is below $1 \mathrm{~mA} / \mathrm{cm}^{2}$ (Fig. 3c,d). However, Device B with the doping concentration of $1 \%$ shows better performance when the current density is higher than $1 \mathrm{~mA} / \mathrm{cm}^{2}$. The maximum CE of the Device A and Device B are $39.6 \mathrm{~cd} / \mathrm{A}$ and $34.6 \mathrm{~cd} / \mathrm{A}$, respectively, which is significantly improved compared to OLED device employing conventional fluorescent material tris(8-hydroxyquinolinolato) aluminum $\left(\mathrm{Alq}_{3}\right)$ as host for $\mathrm{c} 545 \mathrm{t}(\sim 12.8 \mathrm{~cd} / \mathrm{A})^{14}$. For yellow OLED devices based on rubrene, Device $\mathrm{D}$ with the doping concentration of $1 \%$ shows the maximum performances of $34.5 \mathrm{~cd} / \mathrm{A}, 41.6 \mathrm{~lm} / \mathrm{W}$ and $10.0 \%$, which is much higher than OLED device using only rubrene as the EML $(\sim 0.4 \mathrm{~lm} / \mathrm{W})$ and OLED device based on rubrene doped with 4,4'-bis[N-(1-napthyl)-N-phenylamion] biphenyl ( $\alpha$-NPD) and $\mathrm{Alq}_{3}(\sim 5.9 \mathrm{~lm} / \mathrm{W})^{20,21}$. The summary of performances of the OLED devices is shown in Table 2.

In conclusion, we have developed highly efficient green and yellow OLED device based on TADF host with a small $\Delta \mathrm{E}_{\mathrm{ST}}$ of $27 \mathrm{meV}$. By using the TADF host and conventional fluorescent emitter c545t and rubrene as the emissive layer, greater efficient energy transfers between the exciplex and the dopants improves device performance significantly. The high performances are result from the efficient utilization of triplet excitons generated in the TADF host through Forster transfer excite the dopants. The OLED devices show excellent performances, which are greatly improved compared to conventional fluorescent OLED devices.

\section{Methods}

The PL spectra were measured by exciting the sample with a $325 \mathrm{~nm}$ He-Cd laser. The absorption spectra were measured by HP 8453 UV-visible spectrophotometer. The time-resolved PL were obtained using an oscilloscope (Agilent Infiniium) and samples were excited with a pulsed $355 \mathrm{~nm}$ Nd:YAG laser. Different ambient temperatures of the sample were realized using EDWARDS Cryodrive 3.0 connected with Intelligent Temperature Controller. 
OLEDs were fabricated by vacuum thermal evaporation under the base pressure $\sim 4 \times 10^{-6}$ Torr. The active area of the OLEDs is $10.89 \mathrm{~mm}^{2}$. All the materials were used as purchased. The luminance, EL spectrum and current density versus voltage $(\mathrm{J}-\mathrm{V})$ characteristics of the OLED device were measured using Spectrascan 650 spectrometer connected with Keithley 236 source measure unit under room temperature. The EQE was calculated from spectral luminance intensity in the forward angle direction of the OLED device with a Lambertian-pattern assumption.

Data availability. The datasets generated and/or analyzed during the current study are available from the corresponding author on reasonable request.

\section{References}

1. Adachi, C., Baldo, M. A., Thompson, M. E. \& Forrest, S. R. Nearly 100\% internal phosphorescence efficiency in an organic lightemitting device. J. Appl. Phys. 90, 5048-5051 (2001).

2. Uoyama, H., Goushi, K., Shizu, K., Nomura, H. \& Adachi, C. Highly efficient organic light-emitting diodes from delayed fluorescence. Nature 492, 234-238 (2012).

3. Jankus, V. et al. Highly efficient TADF OLEDs: how the emitter-host interaction controls both the excited state species and electrical properties of the devices to achieve near 100\% triplet harvesting and high efficiency. Adv. Funct. Mater. 24, 6178-6186 (2014).

4. Baleizão, C. \& Berberan-Santos, M. N. Thermally activated delayed fluorescence as a cycling process between excited singlet and triplet states: Application to the fullerene. J. Chem. Phys. 126, 204510 (2007).

5. Schwartz, G., Reineke, S., Rosenow, T. C., Walzer, K. \& Leo, K. Triplet harvesting in hybrid white organic light-emitting diodes. Adv. Funct. Mater. 19, 1319-1333 (2009).

6. Endo, A. et al. Efficient up-conversion of triplet excitons into a singlet state and its application for organic light emitting diodes. Appl. Phys. Lett. 98, 083302 (2011).

7. Yan, F., Chen, R., Sun, H. \& Sun, X. W. Organic light-emitting diodes with a spacer enhanced exciplex emission. Appl. Phys. Lett. 104, $153302(2014)$.

8. Chen, H. F., Liao, C. T., Su, H. C., Yeh, Y. S. \& Wong, K. T. Highly efficient exciplex emission in solid-state light-emitting electrochemical cells based on mixed ionic hole-transport triarylamine and ionic electron-transport 1,3,5-triazine derivatives. J. Mater. Chem. C 1, 4647-4654 (2013).

9. Zhang, T. et al. ACS Appl. Mater. Interfaces 6, 11907-11914 (2014).

10. Zhao, B. et al. Highly efficient red OLEDs using DCJTB as the dopant and delayed fluorescent exciplex as the host. Sci. Rep 5, 10697 (2015).

11. Zhang, L. et al. Efficient organic light emitting diode through triplet exciton reharvesting by employing blended electron donor and acceptor as the emissive layer. ACS Appl. Mater. Interfaces 7, 24983-24986 (2015).

12. Dias, F. B., Penfold, T. J. \& Monkman, A. P. Photophysics of thermally activated delayed fluorescence molecules. Methods Appl. Fluoresc. 5, 012001 (2017).

13. Dias, F. B. et al. The role of local triplet excited states and D-A relative orientation in thermally activated delayed fluorescence: photophysics and devices. Adv. Sci. 3, 1600080 (2016).

14. Liu, Z., Helander, M. G., Wang, Z. \& Lu, Z. Efficient single-layer organic light-emitting diodes based on C545T-Alq3 system. J. Phys. Chem. C 114, 11931-11935 (2010).

15. Chang, M. Y. et al. High-color-purity organic light-emitting diodes incorporating a cyanocoumarin-derived red dopant material. J. Electrochem. Soc. 155, J365-J370 (2008).

16. Liu, X. K. et al. Energy transfer: nearly $100 \%$ triplet harvesting in conventional fluorescent dopant-based organic light-emitting devices through energy transfer from exciplex. Adv. Mater. 27, 2025 (2015).

17. Kim, W. Y. et al. Spectroscopic study of white organic light-emitting devices with various thicknesses of emissive layer. J. Appl. Phys. 111, 014507 (2012).

18. Limketkai, B. N. \& Baldo, M. A. Charge injection into cathode-doped amorphous organic semiconductors. Phys. Rev. B 71, 085207 (2005).

19. Malliaras, G. G., Salem, J. R., Brock, P. J. \& Scott, C. Electrical characteristics and efficiency of single-layer organic light-emitting diodes. Phys. Rev. B 58, R13411 (1998).

20. Okumoto, K., Kanno, H., Hamada, Y., Takahashi, H. \& Shibata, K. High efficiency red organic light-emitting devices using tetraphenyldibenzoperiflanthene-doped rubrene as an emitting layer. Appl. Phys. Lett. 89, 013502 (2006).

21. Wang, Z., Naka, S. \& Okada, H. Performance improvement of rubrene-based organic light emitting devices with a mixed single layer. Appl. Phys. A 100, 1103-1108 (2010).

\section{Author Contributions}

L.Z. performed the experiments. K.W.C. on discussion and analysis. All authors wrote the manuscript and discussed the results.

\section{Additional Information}

Supplementary information accompanies this paper at https://doi.org/10.1038/s41598-018-27238-y.

Competing Interests: The authors declare no competing interests.

Publisher's note: Springer Nature remains neutral with regard to jurisdictional claims in published maps and institutional affiliations.

Open Access This article is licensed under a Creative Commons Attribution 4.0 International License, which permits use, sharing, adaptation, distribution and reproduction in any medium or format, as long as you give appropriate credit to the original author(s) and the source, provide a link to the Creative Commons license, and indicate if changes were made. The images or other third party material in this article are included in the article's Creative Commons license, unless indicated otherwise in a credit line to the material. If material is not included in the article's Creative Commons license and your intended use is not permitted by statutory regulation or exceeds the permitted use, you will need to obtain permission directly from the copyright holder. To view a copy of this license, visit http://creativecommons.org/licenses/by/4.0/.

(C) The Author(s) 2018 